\title{
Determination of Anionic Detergent Concentration of Karasu Stream in Sinop (Turkey)
}

\author{
Ayşe Gündoğdu ${ }^{*}$, Erdi Gültepe ${ }^{2}$, Uğur Çarlı ${ }^{3}$ \\ ${ }^{1}$ Fisheries Faculty, Sinop University, 57000 Sinop, Turkey \\ ${ }^{2}$ Private sector, 38020 Kayseri, Turkey \\ ${ }^{3}$ Scientific and Technological Researchs, Application and Research Center, Sinop University, 57000 Sinop, Turkey
}

\section{A R T I C L E I N F O}

\section{Research Articles}

Received 24 October 2017

Accepted 20 December 2017

\section{Keywords:}

\section{Surfactants}

Fresh water

Physico-chemical

River

Creek

Black Sea

\section{A B S T R A C T}

The study was achieved between May 2014 and April 2015 at the Karasu Creek located in the province of Sinop. It was conducted to determine anionic detergent pollution and some physicochemical properties $(\mathrm{pH}$, temperature, conductivity, salinity, dissolved oxygen, total hardness, chemical oxygen demand, phosphate $\mathrm{PO}_{4}^{-3}$, total nitrogen). The anionic detergent concentration of the stations was determined on a monthly basis. Seasonally averaged values of the anionic detergent was measured as the highest value in the autumn season. The lowest values of anionic detergent were found in stations in winter and spring. The increase in the concentration of anionic detergent is caused by population growth in residential areas, increased agricultural activities and rains, and that chemicals move to riverbed from terrestrial areas with rain water.

${ }^{*}$ Corresponding Author:

E-mail: aysegundogdu57@hotmail.com

Türk Tarım - Gıda Bilim ve Teknoloji Dergisi, 6(1): 112-123, 2018

\section{Sinop'ta Bulunan Karasu Deresi'nin Anyonik Deterjan Konsantrasyonunun Belirlenmesi (Türkiye)}

M A K A L E B İ L G İ S İ

\section{AraştırmaMmakalesi}

Geliş 24 Ekim 2017

Kabul 20 Aralık 2017

Anahtar Kelimeler:

Yüzey aktif maddeler

$\mathrm{Su}$

Fiziko-kimyasal

Nehir

Dere

Karadeniz

\section{Ö Z E T}

Çalışma, Mayıs 2014 - Nisan 2015 tarihleri arasında Sinop il sınırları içerisinde bulunan Karasu Deresi'nde gerçekleştirilmiştir. Derenin Anyonik deterjan kirliliği ve bazı fiziko kimyasal parametrelerinin $(\mathrm{pH}$, sicaklık, iletkenlik, tuzluluk, çözünmüş oksijen, toplam sertlik, kimyasal oksijen ihtiyac1, fosfat $\mathrm{PO}_{4}^{-3}$, toplam azot) değerlendirilmesi amaçlanmıştır. Belirlenen istasyonlarda Anyonik deterjan konsantrasyonu aylık olarak ölçülmüştür. Ölçüm sonuçları mevsimsel olarak değerlendirildiğinde en yüksek değerin Sonbahar mevsiminde, en düşük değerlerin ise kış ve ilkbahar mevsiminde olduğu tespit edilmiştir. Anyonik deterjan konsantrasyonundaki değişim, yerleşim yerlerindeki nüfus artışı, tarımsal faaliyetlerin yoğunlaşması ve artan yağışlar nedeniyle kimyasalların karasal alanlardan nehir alanlarına geçmesinden kaynaklanmaktadır.

*Sorumlu Yazar:

E-mail: aysegundogdu57@hotmail.com 


\section{Introduction}

The need for water resources in Turkey is steadily increasing as is the case in the world, and unfortunately the negative environmental effects on limited resources are getting increasingly intense. The dams and lakes that meet the need for drinking and utility water, as well as domestic and industrial wastes, are under the pressure of intensive construction in residential areas. Rivers, lakes, underground waters, and seas being in-continental water resources, are adversely affected by domestic and industrial wastes, excessive fertilizers and unconscious pesticides. In developing countries today, $80 \%$ of all diseases and one third (1/3) of deaths are caused by contaminated water (İleri, 2000; Bayram and Önsoy, 2011).

Detergents are often used in washing agents, dishwashing agents, and shampoos thanks to their high foaming and cleansing abilities. These detergents are also used in textile fabrics. So they are more likely to get mixed into natural waters as they are found in many types of wastes. The most commonly used surfactants are anionic surfactants, and represent about $50 \%$ of the world production. Detergent contamination is important because it affects the biological activity in the water. Toxic effects are generally reported to occur in organisms when the amount of detergent in sea water is higher than $0.1 \mathrm{~g} / \mathrm{m}^{3}$ (Egemen, 2000). These effects vary by the environmental conditions and the tolerance of the species, many of which are shown with different lethal dose values.

The amount of detergent, nitrogen and phosphorus that comes with domestic and industrial wastewater, and through drainage and rainwater from agricultural areas, is quite high. Eutrophication stands forefront of the events that adversely affect quality in water resources and lead biodiversity to diminish. The factor which accelerates eutrophication is the increase in nitrogen and phosphorus concentration in the water environment. Eutrophication is regarded to be the limit value when the phosphorus concentration exceeds $0.1 \mathrm{mg} / \mathrm{L}$ in given water source (Egemen, 2000). It has been reported that phosphorus, which is effective in the growth of algae, comes from only $10 \%$ detergents, from $16 \%$ fertilizers made in agricultural areas, and the rest comes from $74 \%$ other uses (Morse et al. 1993; Kundu et al., 2015). Detergents are not only effective in the water system due to the increase in solubility or concentration, but they also undergo biodegradation causing changes in water properties.

When nutrients (nitrogen and phosphorus) reach water sources, they cause a multiplication of algae and an increase in the amount of organic matter. The excessive increase of the algae causes a decrease in the dissolved oxygen in the water and image pollution raises due to the excess of algae (Gündoğdu and Erdem, 1995). In addition, biomass to mass death is caused by oxygen deficiency, competition between some algae species and secretion of toxic substances. As a result, it is likely that nitrogen and phosphorus will be released again, including this cycle, since the algae are involved in the disintegration and biochemical transformations.

The environment is affected by non-conforming effects and responds to pollution factors with new behaviors (bacteria, enzymes) to redress the balance again. Microorganisms can act very quickly in harmony with deteriorated environmental conditions. Some of the microorganisms in the water can be adapted to detergents while some cannot. However, a certain period of time is required to complete the degradation under appropriate conditions. The adaptation of advanced organisms to deteriorated environmental conditions takes long time. It can be said that the reactions to the detergents used as cleansing and washing substances of the organisms are similar. Microorganisms use detergents and exchange them with non-simple chemical events. Detergents are consumed by microorganisms, that is, they are eaten as food. While the formerly used tetrapropylene benzene sulphonate has been degraded by $30 \%$, currently used detergents are degraded by $90 \%$ (Minareci, 2007).

Biodegradation is the process of separating organic compounds into small pieces by means of using enzymes through microbial organisms. Factors responsible for the biodegradation of detergents are as follows. The number of detergent active ingredients, detergent concentration, the amount of organic matter in the environment and its composition, mineral levels, the adaptation grades and nature of available microorganisms, $\mathrm{pH}$ value, temperature, duration of exposure, ventilation of the environment. In a study conducted on the biodegradation of anionic detergents, Achromobacter, Klebsiella, Micrococcus and Flavobacterium members were reported to degrade detergents. It was also suggested that the degradation is dependent on the environmental conditions and is affected or inhibited by adverse conditions. Gravel, asbestos or dried in the presence of activated sludge was found to have degradation. It has been determined that chemicals such as peptone inhibit degradation (Cook, 1968). In the present study, some physicochemical properties and anionic detergent level of Karasu stream were evaluated for the first time, and the presence environmental effects were investigated.

\section{Materials and Methods}

The study was carried out between May 2014 and April 2015 at Karasu stream located in the province of Sinop (Black Sea). Sampling of study was performed in monthly periods. It was conducted to determine anionic detergent pollution and some physicochemical properties [pH, temperature, conductivity, salinity, dissolved oxygen (DO), total hardness, chemical oxygen demand (COD), phosphorus (P) and total nitrogen (TN)] of the water quality of Karasu stream.

\section{Characteristics of The Research Area}

Characteristics of Karasu Stream: Karasu Stream is born from Gündüzlü Forests in Küre Mountains in Black Sea Region. It extends to Erfelek district acting in southnorth direction, and it empties its waters into the Black Sea from $8 \mathrm{~km}$ west of Sinop province. The length of the stream is about $80 \mathrm{~km}$ and it moves in a broad-bottomed valley. Çatak, Kınık, Çakçak, Hasan, Ramlı and Tasnak creeks are feeding Karasu stream (Çarlı, 2015). Erfelek waterfall is located within the boundaries of Tatlica village of Karasu Stream. It is constituted by 28 cascades 
which are arranged in the form of stair steps along the valley (Uzun, et al., 2005). Drinking water treatment plant of Sinop province supplies water from the waterfall located in the direction of Karasu stream. In the study, samples were taken from four different stations determined on Karasu stream. Particularly, stations were identified taking discharge points into account. Station K1: The bridge of Karasu stream is located in Akliman settlement area in Sinop city center, and under the influence of the region. It is the area where Karasu stream merges with the sea. Station K2 (Akkent site residential area): The station is behind Akkent Site residential area. However, there has been a recent development in tourism in Akliman area of Sinop. This region is located within the tourist residential area and the proximity of the Sinop central villages is a cause of intense human activities. Station K3 (Karasu bridge): Karasu bridge is located in the village of Dibekli on the way to Ayancık. The reason for selecting the station; the density of the settlement area and agricultural land is high, and hospital wastes are likely to interfere with Karasu stream. For this reason, Station K3 is an important area in determining whether environmental effects are present or not. Station K4 (Mertoğlu village): Mertoglu village is located $10 \mathrm{~km}$ away from the center of Sinop. An important factor in choosing this station is intense agricultural activities carried out here. The possibility of reaching Karasu river with the irrigation channels and rain water of the chemicals used in agriculture was taken into consideration.

\section{Physicochemical Parameters}

Measurements of $\mathrm{pH}$ value, temperature, conductivity, DO values were instantly taken by YSI Multiparameter (Professsion Plus model) device. Other physicochemical parameters [Salinity, COD, total hardness (by titrimetric method); ammonia nitrogen $\left(\mathrm{NH}_{4}-\mathrm{N}\right)$, nitrate nitrogen $\left(\mathrm{NO}_{3}-\mathrm{N}\right)$, nitrite nitrogen $\left(\mathrm{NO}_{2}-\mathrm{N}\right)$, phosphate phosphorus $\left(\mathrm{PO}_{4}-\mathrm{P}\right) \mathrm{mg} / \mathrm{L}$ (by spectrophotometric method)] for wastewater characterisation measurement are performed in accordance with Standard Methods (TSI, 2005; APHA, 1965; Egemen and Sunlu, 1996).The total nitrogen was calculated over the concentration of ammonium, nitrite and nitrate (respectively, $\mathrm{NH}_{4}+\mathrm{NO}_{2}+\mathrm{NO}_{3}$ ).

\section{Anionic Detergent Process}

The colorimetric (methylene blue) method was used to quantify the total concentrations of anionic surfactant as methylene blue active substances (MBAS) (Leithe, 1973). Sea water was shaken with a mixture of phosphate buffer $\left(\mathrm{Na}_{2} \mathrm{HPO}_{4} ; \quad \mathrm{pH}=10\right)$, neutral methylene blue and chloroform. Then, the separated chloroform layer was shaken with distilled water and acidic methylene blue solution in a second separatory funnel. The extraction from acidic aqueous medium into chloroform was repeated two more times by adding chloroform. The chloroform phase obtained from the acidic solution was filtered through a glass cotton and the balloon was collected in the vial. The intensity of the acquired blue color, which was proportional to the concentration of the extracted Anionic Surfactant in the organic phase, was measured by spectrophotometer at $652 \mathrm{~nm}$ (Shimadzu, UV-1800). The acquired intensity of the blue color was proportional to the anionic surfactant concentration in the extracted organic phase. Calibration graph was drawn using standard solutions of sodium sulfosuccinic acid dioctyl ester $\left(\mathrm{C}_{20} \mathrm{H}_{37} \mathrm{NaO}_{7} \mathrm{~S}\right)$, and concentrations of the samples were calculated (APHA, 1981; Egemen, and Sunlu, 1996).

Statistical Analysis

Statistical analyzes of water quality parameters were calculated with SPSS 22 and Minitab 13 program. All parameters were also conducted two-way analysis of variance (Anova test). In addition, correlation matrices were created to evaluate the relationships between the examined physicochemical concentrations of the samples (Table 5). The values of correlation coefficients between physicochemical parameters in months were also given statistically significant if $\mathrm{P} \leq 0.01$ and $\mathrm{P} \leq 0.001$ (except $\mathrm{P} \geq 0.05$ ).

\section{Results and Discussions}

Anionic detergent and some physicochemical parameters were selected to determine the quality of water in Karasu stream. The classification of the stream was made according to Classes of Quality Criteria of Continental Water Resources of the Water Pollution Control Regulation (SWQR, 2015). The results of the measurement and analysis of anionic detergent, $\mathrm{pH}$ value, temperature, conductivity, salinity, DO, total hardness, KOD, $\mathrm{P}$ and TN obtained from the study are presented. Whether there is statistical significance between the groups is shown in Tables. There were significant differences $(\mathrm{P}<0.05)$ between average values when both months and stations were compared within themselves (Table 1-4). Although the mean values of $\mathrm{pH}$, temperature, conductivity, salinity and phosphorus were significantly different when compared within months $(\mathrm{P}<0.05)$, there was no significant difference $(\mathrm{P}>0.05)$ between the stations (Table 1 and 2). Both months and stations were significant differences $(\mathrm{P}<0.05)$ between the mean values (DO, TH, COD, TN) compared to their own internal (Table 2 and 3). Anionic detergent concentrations were statistically significant differences in months $(\mathrm{P}<0.05)$, there was no significant difference $(\mathrm{P}>0.05)$ between the stations (Table 4 ).

The average annual $\mathrm{pH}$ value of the stream was determined to be 7.41 (min-max.: 6.68-8.12). When seasonal $\mathrm{pH}$ changes are examined, it can be said that their water is close to neutral and basic character (Table 1). Some monthly $\mathrm{pH}$ differences may be attributed to changes in phytoplankton and $\mathrm{CO}_{2}$. Studies conducted on river waters in different regions have shown that $\mathrm{pH}$ values are in parallel with the findings of the present study (Table 5). One of the important parameters affecting other parameters in water quality is temperature. The temperature tends to form a meaningful coherence, especially with other parameters such as dissolved oxygen, biological oxygen demand and so on (Gürel, 2011). The temperature values of Karasu stream were found to vary between $10.06-27.13^{\circ} \mathrm{C}$. When studying the determined temperature values, it was found that water source is higher in summer. Seasonal changes in air temperature can be considered to have an effect on this situation. It is known that the temperature value of water resources varies depending on climate, altitude, water supply, flow rate and bed structure, and atmospheric conditions (Cirik and Cirik, 2008). 
Table 1 Monthly mean and standard deviation $(\mathrm{X} \pm \mathrm{SD})$ values for $\mathrm{pH}$, Temperature, Conductivity, Salinity of water sample in Karasu stream $(\mathrm{n}=3)$.

\begin{tabular}{|c|c|c|c|c|}
\hline \multirow{2}{*}{ Months } & \multicolumn{4}{|c|}{ Stations } \\
\hline & $\mathrm{K} 1$ & $\mathrm{~K} 2$ & K3 & K4 \\
\hline \multicolumn{5}{|c|}{$\mathrm{pH}$} \\
\hline May & $6.76 \pm 0.01$ & $6.75 \pm 0.01$ & $6.68 \pm 0.01$ & $6.97 \pm 0.01$ \\
\hline June & $7.65 \pm 0.01$ & $7.57 \pm 0.01$ & $7.77 \pm 0.02$ & $7.94 \pm 0.02$ \\
\hline July & $7.79 \pm 0.02$ & $7.82 \pm 0.02$ & $7.31 \pm 0.01$ & $7.54 \pm 0.01$ \\
\hline August & $8.12 \pm 0.02$ & $7.72 \pm 0.06$ & $7.87 \pm 0.02$ & $7.95 \pm 0.02$ \\
\hline September & $7.64 \pm 0.02$ & $7.68 \pm 0.02$ & $7.74 \pm 0.04$ & $7.69 \pm 0.01$ \\
\hline October & $7.49 \pm 0.02$ & $7.61 \pm 0.01$ & $7.63 \pm 0.01$ & $7.33 \pm 0.00$ \\
\hline November & $7.24 \pm 0.01$ & $7.35 \pm 0.01$ & $7.28 \pm 0.01$ & $7.20 \pm 0.01$ \\
\hline December & $7.09 \pm 0.01$ & $7.12 \pm 0.02$ & $7.07 \pm 0.01$ & $7.13 \pm 0.02$ \\
\hline January & $7.10 \pm 0.02$ & $7.08 \pm 0.01$ & $7.04 \pm 0.01$ & $7.11 \pm 0.01$ \\
\hline February & $7.19 \pm 0.01$ & $7.21 \pm 0.02$ & $7.27 \pm 0.02$ & $7.21 \pm 0.01$ \\
\hline March & $7.41 \pm 0.01$ & $7.36 \pm 0.02$ & $7.46 \pm 0.01$ & $7.46 \pm 0.02$ \\
\hline April & $7.72 \pm 0.02$ & $7.62 \pm 0.02$ & $7.57 \pm 0.01$ & $7.70 \pm 0.01$ \\
\hline \multicolumn{5}{|c|}{ Temperature $\left({ }^{\circ} \mathrm{C}\right)$} \\
\hline May & $18.13 \pm 0.15$ & $18.08 \pm 0.02$ & $17.43 \pm 0.03$ & $17.55 \pm 0.05$ \\
\hline June & $23.25 \pm 0.09$ & $23.09 \pm 0.08$ & $21.70 \pm 0.03$ & $20.69 \pm 0.10$ \\
\hline July & $26.04 \pm 0.11$ & $25.83 \pm 0.06$ & $20.95 \pm 0.06$ & $21.56 \pm 0.08$ \\
\hline August & $27.13 \pm 0.03$ & $26.78 \pm 0.09$ & $25.70 \pm 0.10$ & $26.25 \pm 0.05$ \\
\hline September & $16.15 \pm 0.05$ & $16.50 \pm 0.10$ & $16.16 \pm 0.05$ & $16.38 \pm 0.07$ \\
\hline October & $12.21 \pm 0.05$ & $12.10 \pm 0.04$ & $12.60 \pm 0.06$ & $12.45 \pm 0.06$ \\
\hline November & $10.06 \pm 0.04$ & $10.16 \pm 0.04$ & $10.22 \pm 0.03$ & $10.19 \pm 0.06$ \\
\hline December & $10.72 \pm 0.06$ & $10.78 \pm 0.09$ & $10.32 \pm 0.04$ & $10.10 \pm 0.04$ \\
\hline January & $10.16 \pm 0.02$ & $11.21 \pm 0.03$ & $11.08 \pm 0.09$ & $11.12 \pm 0.11$ \\
\hline February & $13.21 \pm 0.02$ & $13.10 \pm 0.05$ & $13.15 \pm 0.01$ & $13.27 \pm 0.05$ \\
\hline March & $15.41 \pm 0.02$ & $15.29 \pm 0.06$ & $15.60 \pm 0.06$ & $16.35 \pm 0.05$ \\
\hline April & $16.11 \pm 0.02$ & $16.21 \pm 0.05$ & $17.07 \pm 0.08$ & $17.32 \pm 0.05$ \\
\hline \multicolumn{5}{|c|}{ Conductivity $(\mu \mathrm{s} / \mathrm{cm})$} \\
\hline May & $1.72 \pm 0.02$ & $1.22 \pm 0.05$ & $0.80 \pm 0.02$ & $0.80-0.85$ \\
\hline June & $3.06 \pm 0.02$ & $2.89 \pm 0.05$ & $2.20 \pm 0.10$ & $1.64 \pm 0.02$ \\
\hline July & $28.83 \pm 0.04$ & $20.47 \pm 0.03$ & $3.44 \pm 0.04$ & $2.63 \pm 0.02$ \\
\hline August & $7.57 \pm 0.02$ & $12.08 \pm 0.06$ & $3.32 \pm 0.02$ & $3.09 \pm 0.04$ \\
\hline September & $8.30 \pm 0.05$ & $8.32 \pm 0.03$ & $44.17 \pm 0.11$ & $44.31 \pm 0.04$ \\
\hline October & $22.08 \pm 0.04$ & $12.74 \pm 0.18$ & $8.81 \pm 0.07$ & $3.77 \pm 0.02$ \\
\hline November & $21.23 \pm 0.04$ & $10.43 \pm 0.18$ & $6.84 \pm 0.04$ & $7.63 \pm 0.03$ \\
\hline December & $20.56 \pm 0.04$ & $9.85 \pm 0.03$ & $6.11 \pm 0.03$ & $7.08 \pm 0.02$ \\
\hline January & $19.73 \pm 0.03$ & $9.11 \pm 0.01$ & $5.73 \pm 0.03$ & $6.10 \pm 0.02$ \\
\hline February & $19.07 \pm 0.05$ & $8.32 \pm 0.03$ & $4.52 \pm 0.03$ & $5.26 \pm 0.05$ \\
\hline March & $22.77 \pm 0.02$ & $20.50 \pm 0.01$ & $13.64 \pm 0.04$ & $14.41 \pm 0.02$ \\
\hline April & $24.54 \pm 0.04$ & $24.50 \pm 0.07$ & $18.20 \pm 0.03$ & $35.67 \pm 0.03$ \\
\hline \multicolumn{5}{|c|}{ Salinity $(\mathrm{g} / \mathrm{L})$} \\
\hline May & $0.029 \pm 0.004$ & $0.023 \pm 0.005$ & $0.015 \pm 0.003$ & $0.013 \pm 0.003$ \\
\hline June & $0.036 \pm 0.002$ & $0.035 \pm 0.005$ & $0.027 \pm 0.003$ & $0.029 \pm 0.005$ \\
\hline July & $0.037 \pm 0.001$ & $0.297 \pm 0.023$ & $0.035 \pm 0.011$ & $0.040 \pm 0.005$ \\
\hline August & $0.097 \pm 0.024$ & $0.153 \pm 0.008$ & $0.060 \pm 0.008$ & $0.052 \pm 0.005$ \\
\hline September & $0.175 \pm 0.058$ & $0.273 \pm 0.027$ & $0.058 \pm 0.001$ & $0.057 \pm 0.001$ \\
\hline October & $0.272 \pm 0.033$ & $0.116 \pm 0.058$ & $0.116 \pm 0.001$ & $0.068 \pm 0.010$ \\
\hline November & $0.276 \pm 0.005$ & $0.140 \pm 0.030$ & $0.099 \pm 0.011$ & $0.099 \pm 0.005$ \\
\hline December & $0.270 \pm 0.010$ & $0.130 \pm 0.030$ & $0.087 \pm 0.011$ & $0.087 \pm 0.005$ \\
\hline January & $0.256 \pm 0.005$ & $0.120 \pm 0.030$ & $0.076 \pm 0.012$ & $0.076 \pm 0.005$ \\
\hline February & $0.243 \pm 0.005$ & $0.110 \pm 0.030$ & $0.064 \pm 0.012$ & $0.064 \pm 0.005$ \\
\hline March & $0.300 \pm 0.010$ & $0.270 \pm 0.010$ & $0.181 \pm 0.006$ & $0.187 \pm 0.006$ \\
\hline April & $0.316 \pm 0.005$ & $0.316 \pm 0.005$ & $0.239 \pm 0.005$ & $0.477 \pm 0.008$ \\
\hline
\end{tabular}

K1, K2, K3, K4 expressions Karasu Stream Stations; (among stations P>0.05), among months ( $\mathrm{P}<0.05$ ). 
Table 2 Monthly mean and standard deviation $(\mathrm{X} \pm \mathrm{SD})$ values for DO, Total Hardness, COD and $\mathrm{P}$ of water sample in Karasu stream.

\begin{tabular}{|c|c|c|c|c|}
\hline \multirow{2}{*}{ Months } & \multicolumn{4}{|c|}{ Stations } \\
\hline & K1 & $\mathrm{K} 2$ & K3 & K4 \\
\hline \multicolumn{5}{|c|}{$\mathrm{DO}(\mathrm{mg} / \mathrm{L})$} \\
\hline May & $5.40 \pm 0.20$ & $5.00 \pm 0.20$ & $5.40 \pm 0.20$ & $5.53 \pm 0.30$ \\
\hline June & $2.40 \pm 0.20$ & $2.66 \pm 0.23$ & $4.66 \pm 0.11$ & $4.93 \pm 0.50$ \\
\hline July & $2.73 \pm 0.11$ & $2.33 \pm 0.11$ & $3.23 \pm 0.15$ & $3.73 \pm 0.30$ \\
\hline August & $3.06 \pm 0.30$ & $5.40 \pm 0.20$ & $3.60 \pm 0.20$ & $5.26 \pm 0.30$ \\
\hline September & $4.80 \pm 0.20$ & $5.53 \pm 0.23$ & $4.86 \pm 0.23$ & $5.53 \pm 0.11$ \\
\hline October & $5.60 \pm 0.20$ & $5.66 \pm 0.11$ & $5.86 \pm 0.11$ & $5.73 \pm 0.11$ \\
\hline November & $6.23 \pm 0.25$ & $6.16 \pm 0.20$ & $6.50 \pm 0.10$ & $6.36 \pm 0.05$ \\
\hline December & $6.40 \pm 0.26$ & $6.26 \pm 0.20$ & $6.63 \pm 0.15$ & $6.56 \pm 0.05$ \\
\hline January & $6.56 \pm 0.20$ & $6.43 \pm 0.15$ & $6.80 \pm 0.10$ & $6.73 \pm 0.05$ \\
\hline February & $6.86 \pm 0.20$ & $6.73 \pm 0.15$ & $7.10 \pm 0.10$ & $7.03 \pm 0.06$ \\
\hline March & $6.33 \pm 0.25$ & $6.16 \pm 0.16$ & $6.46 \pm 0.15$ & $6.50 \pm 0.10$ \\
\hline April & $5.76 \pm 0.15$ & $5.56 \pm 0.15$ & $5.60 \pm 0.20$ & $5.83 \pm 0.11$ \\
\hline \multicolumn{5}{|c|}{ Total Hardness (mg/L) } \\
\hline May & $226.83 \pm 1.25$ & $236.96 \pm 1.00$ & $232.16 \pm 1.25$ & $212.66 \pm 1.15$ \\
\hline June & $230.23 \pm 1.56$ & $242.03 \pm 0.57$ & $239.13 \pm 1.02$ & $245.16 \pm 1.04$ \\
\hline July & $241.70 \pm 0.60$ & $258.66 \pm 1.25$ & $429.10 \pm 1.01$ & $369.30 \pm 1.12$ \\
\hline August & $66.26 \pm 1.61$ & $61.83 \pm 0.72$ & $56.66 \pm 1.52$ & $57.66 \pm 1.52$ \\
\hline September & $41.66 \pm 1.52$ & $47.80 \pm 0.57$ & $37.43 \pm 1.40$ & $31.73 \pm 1.41$ \\
\hline October & $36.26 \pm 1.61$ & $41.33 \pm 1.61$ & $30.36 \pm 0.63$ & $23.33 \pm 0.57$ \\
\hline November & $37.33 \pm 0.57$ & $41.73 \pm 1.67$ & $30.33 \pm 0.57$ & $24.13 \pm 1.02$ \\
\hline December & $37.26 \pm 1.41$ & $39.93 \pm 1.52$ & $27.70 \pm 1.57$ & $21.33 \pm 1.52$ \\
\hline January & $33.20 \pm 1.31$ & $37.66 \pm 1.57$ & $25.33 \pm 0.57$ & $21.26 \pm 1.61$ \\
\hline February & $25.66 \pm 1.52$ & $27.70 \pm 1.41$ & $14.40 \pm 1.50$ & $10.10 \pm 0.85$ \\
\hline March & $186.06 \pm 0.90$ & $204.26 \pm 1.00$ & $201.80 \pm 1.31$ & $214.06 \pm 1.10$ \\
\hline April & $326.10 \pm 1.01$ & $339.00 \pm 1.00$ & $304.40 \pm 0.52$ & $353.66 \pm 1.52$ \\
\hline \multicolumn{5}{|c|}{$\mathrm{COD}(\mathrm{mg} / \mathrm{L})$} \\
\hline May & $8.77 \pm 0.37$ & $7.49 \pm 0.37$ & $9.88 \pm 0.43$ & $11.98 \pm 0.98$ \\
\hline June & $8.18 \pm 0.81$ & $14.41 \pm 0.53$ & $9.61 \pm 0.53$ & $14.06 \pm 0.81$ \\
\hline July & $15.84 \pm 0.66$ & $18.26 \pm 0.76$ & $16.06 \pm 0.76$ & $18.26 \pm 0.38$ \\
\hline August & $18.06 \pm 0.73$ & $18.28 \pm 0.36$ & $18.92 \pm 0.36$ & $18.49 \pm 0.01$ \\
\hline September & $15.88 \pm 3.41$ & $15.70 \pm 3.28$ & $16.57 \pm 3.60$ & $16.21 \pm 3.36$ \\
\hline October & $20.42 \pm 5.59$ & $19.98 \pm 5.38$ & $21.13 \pm 5.58$ & $20.93 \pm 5.57$ \\
\hline November & $21.35 \pm 2.19$ & $20.92 \pm 2.21$ & $22.12 \pm 2.41$ & $21.89 \pm 2.27$ \\
\hline December & $20.01 \pm 0.63$ & $19.64 \pm 1.18$ & $20.70 \pm 0.87$ & $20.51 \pm 1.01$ \\
\hline January & $18.55 \pm 0.73$ & $18.06 \pm 0.44$ & $18.94 \pm 0.44$ & $19.04 \pm 0.29$ \\
\hline February & $14.69 \pm 2.17$ & $14.16 \pm 1.87$ & $14.23 \pm 1.75$ & $14.90 \pm 2.49$ \\
\hline March & $11.65 \pm 0.84$ & $10.78 \pm 0.58$ & $11.64 \pm 0.60$ & $11.93 \pm 0.85$ \\
\hline April & $7.44 \pm 0.62$ & $6.84 \pm 1.86$ & $12.41 \pm 2.36$ & $14.96 \pm 1.56$ \\
\hline \multicolumn{5}{|c|}{$\mathrm{PO}_{4}^{-3}-\mathrm{P}(\mathrm{mg} / \mathrm{L})$} \\
\hline May & $0.055 \pm 0.003$ & $0.051 \pm 0.002$ & $0.053 \pm 0.004$ & $0.023 \pm 0.001$ \\
\hline June & $0.039 \pm 0.003$ & $0.038 \pm 0.002$ & $0.039 \pm 0.004$ & $0.017 \pm 0.005$ \\
\hline July & $0.024 \pm 0.002$ & $0.035 \pm 0.002$ & $0.027 \pm 0.002$ & $0.012 \pm 0.001$ \\
\hline August & $0.030 \pm 0.003$ & $0.006 \pm 0.001$ & $0.028 \pm 0.001$ & $0.019 \pm 0.002$ \\
\hline September & $0.024 \pm 0.001$ & $0.024 \pm 0.001$ & $0.023 \pm 0.001$ & $0.007 \pm 0.001$ \\
\hline October & $0.030 \pm 0.002$ & $0.040 \pm 0.001$ & $0.017 \pm 0.002$ & $0.010 \pm 0.001$ \\
\hline November & $0.018 \pm 0.001$ & $0.028 \pm 0.001$ & $0.009 \pm 0.001$ & $0.004 \pm 0.001$ \\
\hline December & $0.022 \pm 0.001$ & $0.034 \pm 0.002$ & $0.010 \pm 0.001$ & $0.007 \pm 0.001$ \\
\hline January & $0.021 \pm 0.001$ & $0.033 \pm 0.002$ & $0.009 \pm 0.001$ & $0.005 \pm 0.001$ \\
\hline February & $0.025 \pm 0.001$ & $0.037 \pm 0.001$ & $0.010 \pm 0.001$ & $0.009 \pm 0.001$ \\
\hline March & $0.043 \pm 0.002$ & $0.042 \pm 0.001$ & $0.031 \pm 0.001$ & $0.013 \pm 0.001$ \\
\hline April & $0.038 \pm 0.004$ & $0.047 \pm 0.002$ & $0.049 \pm 0.004$ & $0.019 \pm 0.001$ \\
\hline
\end{tabular}


Table 3 Monthly mean and standard deviation $(\mathrm{X} \pm \mathrm{SD})$ values for Total $\mathrm{N}\left(\mathrm{NH}_{4}+\mathrm{NO}_{2}+\mathrm{NO}_{3}\right) \mathrm{mg} / \mathrm{L}$ of water sample in Karasu stream.

\begin{tabular}{l|cccc}
\hline \multirow{2}{*}{ Months } & \multicolumn{3}{|c}{ Stations } \\
\cline { 2 - 4 } & K1 & K2 & K3 & K4 \\
\hline May & $0.479 \pm 0.002$ & $0.391 \pm 0.004$ & $0.119 \pm 0.003$ & $0.122 \pm 0.002$ \\
June & $0.630 \pm 0.003$ & $0.681 \pm 0.003$ & $0.185 \pm 0.003$ & $0.221 \pm 0.002$ \\
July & $0.506 \pm 0.004$ & $0.500 \pm 0.004$ & $0.749 \pm 0.003$ & $0.560 \pm 0.003$ \\
August & $0.688 \pm 0.002$ & $0.602 \pm 0.004$ & $0.238 \pm 0.004$ & $0.256 \pm 0.001$ \\
September & $0.486 \pm 0.006$ & $0.414 \pm 0.003$ & $0.272 \pm 0.002$ & $0.226 \pm 0.002$ \\
October & $0.348 \pm 0.008$ & $0.297 \pm 0.004$ & $0.153 \pm 0.003$ & $0.155 \pm 0.002$ \\
November & $0.583 \pm 0.008$ & $0.502 \pm 0.003$ & $0.395 \pm 0.008$ & $0.384 \pm 0.009$ \\
December & $0.653 \pm 0.004$ & $0.589 \pm 0.004$ & $0.479 \pm 0.003$ & $0.425 \pm 0.007$ \\
January & $0.757 \pm 0.005$ & $0.687 \pm 0.004$ & $0.470 \pm 0.007$ & $0.510 \pm 0.005$ \\
February & $0.974 \pm 0.006$ & $0.852 \pm 0.005$ & $0.607 \pm 0.009$ & $0.653 \pm 0.005$ \\
March & $0.763 \pm 0.005$ & $0.788 \pm 0.005$ & $0.432 \pm 0.008$ & $0.505 \pm 0.003$ \\
April & $0.690 \pm 0.007$ & $0.759 \pm 0.006$ & $0.438 \pm 0.006$ & $0.483 \pm 0.004$ \\
\hline K1,K2,K3,K4 exp
\end{tabular}

K1, K2, K3, K4 expressions Karasu Stream Stations; for stations and months $(\mathrm{P}<0.05)$.

Table 4 Monthly mean and standard deviation $(\mathrm{X} \pm \mathrm{SD})$ values of Anionic Detergent $(\mathrm{mg} / \mathrm{L})$ and minimum, maximum values.

\begin{tabular}{|c|c|c|c|c|c|c|c|c|}
\hline \multirow[b]{3}{*}{ Months } & \multicolumn{8}{|c|}{ Stations } \\
\hline & \multicolumn{2}{|c|}{ K1 } & \multicolumn{2}{|c|}{ K2 } & \multicolumn{2}{|c|}{ K3 } & \multicolumn{2}{|c|}{ K4 } \\
\hline & $\mathrm{X} \pm \mathrm{SD}$ & Min-Max & $\mathrm{X} \pm \mathrm{SD}$ & Min-Max & $\mathrm{X} \pm \mathrm{SD}$ & Min-Max & $\mathrm{X} \pm \mathrm{SD}$ & Min-Max \\
\hline May & $0.103 \pm 0.005$ & $0.10-0.11$ & $0.086 \pm 0.015$ & $0.07-0.10$ & $0.093 \pm 0.005$ & $0.09-0.10$ & $0.146 \pm 0.015$ & $0.12-0.15$ \\
\hline June & $0.116 \pm 0.020$ & $0.10-0.14$ & $0.096 \pm 0.015$ & $0.08-0.110$ & $0.103 \pm 0.005$ & $0.10-0.11$ & $0.186 \pm 0.015$ & $0.13-0.16$ \\
\hline July & $0.156 \pm 0.011$ & $0.15-0.17$ & $0.136 \pm 0.015$ & $0.12-0.15$ & $0.143 \pm 0.005$ & $0.14-0.15$ & $0.340 \pm 0.056$ & $0.17-0.20$ \\
\hline August & $0.663 \pm 0.058$ & $0.03-0.99$ & $0.966 \pm 0.015$ & $0.95-0.98$ & $0.973 \pm 0.011$ & $0.96-0.98$ & $0.226 \pm 0.015$ & $0.01-0.99$ \\
\hline September & $0.260 \pm 0.065$ & $0.19-0.32$ & $0.126 \pm 0.047$ & $0.09-0.18$ & $0.126 \pm 0.046$ & $0.10-0.18$ & $0.936 \pm 0.005$ & $0.21-0.24$ \\
\hline October & $0.593 \pm 0.048$ & $0.03-0.90$ & $0.850 \pm 0.052$ & $0.79-0.89$ & $0.883 \pm 0.005$ & $0.88-0.89$ & $0.860 \pm 0.034$ & $0.93-0.94$ \\
\hline November & $0.823 \pm 0.090$ & $0.73-0.91$ & $0.736 \pm 0.058$ & $0.67-0.76$ & $0.876 \pm 0.005$ & $0.87-0.88$ & $0.313 \pm 0.028$ & $0.82-0.88$ \\
\hline December & $0.340 \pm 0.091$ & $0.26-0.44$ & $0.223 \pm 0.073$ & $0.14-0.28$ & $0.333 \pm 0.011$ & $0.32-0.34$ & $0.150 \pm 0.017$ & $0.28-0.33$ \\
\hline January & $0.173 \pm 0.097$ & $0.09-0.28$ & $0.086 \pm 0.032$ & $0.05-0.11$ & $0.170 \pm 0.017$ & $0.15-0.18$ & $0.113 \pm 0.011$ & $0.13-0.16$ \\
\hline February & $0.146 \pm 0.095$ & $0.05-0.24$ & $0.050 \pm 0.026$ & $0.02-0.07$ & $0.133 \pm 0.011$ & $0.12-0.14$ & $0.093 \pm 0.005$ & $0.10-0.12$ \\
\hline March & $0.126 \pm 0.087$ & $0.03-0.15$ & $0.346 \pm 0.053$ & $0.03-0.97$ & $0.130 \pm 0.034$ & $0.09-0.15$ & $0.133 \pm 0.028$ & $0.09-0.10$ \\
\hline April & $0.093 \pm 0.005$ & $0.09-0.10$ & $0.073 \pm 0.037$ & $0.03-0.10$ & $0.193 \pm 0.037$ & $0.15-0.22$ & $0.136 \pm 0.015$ & $0.10-0.15$ \\
\hline
\end{tabular}

K1, K2, K3, K4 expressions Karasu Stream Stations; among stations ( $>>0.05)$, among months ( $\mathrm{P}<0.05)$.

The electrical conductivity of waters depends on the amount and type of salts found in the water, and the temperature of the water (Er, 2014). Conductivity values of Karasu stream were found to be in the range of 0.80 $44.31 \mu \mathrm{S} / \mathrm{cm}$. Electrical conductivity values at stations were not homogeneous. It can be said that the causes of this situation can be very different and the most important reasons are the change of climatic conditions, the connection of the water source to the sea, the changes of temperature and precipitation as well as the existence of other water levers (Table 5). It has been determined that the conductivity values of the stations are different according to the previous studies. Among the reasons why the different results can be displayed, differentiations in season, temperature, water content, soil type, amount of chemical contained, dissolution rate and biological structure can be mentioned.

Salinity is determined through the chloride equivalence in water. The ions that play a role in the formation of salinity are cations $\left(\mathrm{Mg}^{+2}, \mathrm{Ca}^{+2}, \mathrm{~K}^{+}, \mathrm{Na}^{+}\right)$ and anions $\left(\mathrm{CI}^{-}, \mathrm{CO}_{3}^{-}, \mathrm{SO}_{4}^{-2}, \mathrm{HCO}_{3}^{-}\right)$. Besides, the role of $\mathrm{Na}+$ and $\mathrm{Cl}$ - ions in the formation of salinity is of great importance (Yıldı, 2013). The salinity values of Karasu stream were found to be in the range of 0.013-0.477 g/L. When we examined the salinity values at the stations, it was determined that they were fluctuating. The reason for this situation is that the changes in snowfall and precipitation amounts can be explained by the increase in temperature and evaporation.

Among the dissolved gases in water, the amount of oxygen gas is of great importance in terms of water quality. The amount of oxygen gas in water varies depending on temperature, salinity, photosynthetic activities and atmospheric pressure (Gündoğdu, 1995; Boztuğ et al., 2012). In the present study, dissolved oxygen values of Karasu stream were determined at 2.33$7.10 \mathrm{mg} / \mathrm{L}$. When the study results are examined, it can be said that seasonal air and water temperatures are involved in the change of dissolved oxygen values (Table 2 ). As the solubility of gases is inversely proportional to the temperature, the dissolved oxygen value is higher in winter months. The most important reason for the differences between the results of our research and the results of previous studies are regional differences (Table 5). 
Table 5 Comparison of physicochemical parameters in water of different rivers.

\begin{tabular}{|c|c|c|}
\hline Studys & Parameters & References \\
\hline \multicolumn{3}{|c|}{$\mathrm{pH}$} \\
\hline Gediz River & 7.60 & Oner and Celik (2011) \\
\hline Aksu Stream (Giresun) & 7.42 & Şengün (2013) \\
\hline Çanakçı Stream (Giresun) & 7.92 & Dinçer (2014) \\
\hline Karasu River (Sinop) Our research. & 7.41 & This study \\
\hline \multicolumn{3}{|c|}{ Temperature $^{\circ} \mathrm{C}$} \\
\hline Harbiye Kaynak Suyu (Hatay) & $14.7-17.2$ & Tepe and Mutlu (2004) \\
\hline Kestel Stream & $11.31-12.05$ & Bulut et al. (2012) \\
\hline Karasu River (Sinop) & $10.06-27.13$ & This study \\
\hline \multicolumn{3}{|c|}{ Conductivity $\mu \mathrm{s} / \mathrm{cm}$} \\
\hline Sırakaraağaçlar Stream (Sinop) & $0.33-20$ & Bat et al. (2000) \\
\hline Rivers (Trabzon) & $28-450$ & Gültekin et al. (2012) \\
\hline Çiğdem Pond (Kastamonu) & $171.10-280.13$ & Kurnaz et. al.(2016). \\
\hline Karasu River (Sinop) & $0.80-44.31$ & This study \\
\hline \multicolumn{3}{|c|}{ Salinity g/L } \\
\hline Sirakaraağaçlar Stream (Sinop) & $0-9.9(0 \%)$ & Bat et al. (2000) \\
\hline Karanfilliçay Creek (Denizli-Muğla) & $0(0 \%)$ & Bulut et al. (2010) \\
\hline Çinarli Stream (Hafik-Sivas) & $0.01-0.16(0 \%)$ & Mutlu et al. (2016). \\
\hline Karasu River (Sinop) Our research. & $0.013-0.477$ & This study \\
\hline \multicolumn{3}{|c|}{$\mathrm{DO} \mathrm{mg} / \mathrm{L}$} \\
\hline Asi River & $2.6-9.9$ & Taşdemir and Göksu (2001) \\
\hline İyidere (Trabzon) & $9.20-11.50$ & Verep et al. (2005) \\
\hline Karasu River (Sinop) Our research. & $2.33-7.10$ & This study \\
\hline \multicolumn{3}{|c|}{ Total Hardness } \\
\hline Köprüçay River (Antalya) & 223.54 & Çiçek and Ertan (2012) \\
\hline Aksu Stream (Giresun) & 156.47 & Şengün (2013) \\
\hline Karasu River (Sinop) & 130.84 & This study \\
\hline \multicolumn{3}{|c|}{$\mathrm{COD} \mathrm{mg} / \mathrm{L}$} \\
\hline Yenişehir Gölü (Reyhanlı) & $18-41$ & Tepe (2009) \\
\hline Akarçay (Afyonkarahisar-Akşehir) & $7.30-497$ & Kivrak et al. (2012) \\
\hline Sakiz Pond (Kastamonu) & $2.44-5.57$ & Mutlu and Kurnaz (2017) \\
\hline Karasu River (Sinop) Our research. & $6.84-22.12$ & This study \\
\hline \multicolumn{3}{|c|}{$\mathrm{PO}_{4}^{-3}-\mathrm{P} \mathrm{mg} / \mathrm{L}$} \\
\hline Mert River (Samsun) & $0.029-6.116$ & Bakan and Şenel (2000) \\
\hline Asi River & $0.002-2.44$ & Taşdemir and Göksu (2001) \\
\hline Gediz River (Manisa) & $0.0044-0.248\left(\mathrm{~g} / \mathrm{m}^{3}\right)$ & Minareci et al. (2009) \\
\hline Karasu River (Sinop) Our research. & $0.006-0.055$ & This study \\
\hline \multicolumn{3}{|c|}{$\mathrm{TN}\left(\mathrm{NH}_{4}+\mathrm{NO}_{2}+\mathrm{NO}_{3}\right) \mathrm{mg} / \mathrm{L}$} \\
\hline Harşit River (Giresun-Trebolu) & $0.870-2.590$ & Bayram and Önsoy (2011) \\
\hline Karasu River (Sinop) Our research. & $0.122-0.974$ & This study \\
\hline \multicolumn{3}{|c|}{ Anionic Detergent mg/L } \\
\hline İzmir Gulf & $0.032-0.232$ & Yilmaz et al. (2006) \\
\hline Gediz River (Manisa) & $0.084-5.592\left(\mathrm{~g} / \mathrm{m}^{3}\right)$ & Minareci et al. (2009) \\
\hline Harşit River (Giresun-Trebolu) & 0.128 & Bayram and Önsoy (2011) \\
\hline Giresun Coastal Zone & $0.887-1.987$ & Polat and Akkan (2016) \\
\hline Harşit River (Giresun-Trebolu) & $0.311-0.757$ & Akkan (2017) \\
\hline Karasu River (Sinop) Our research. & $0.05-0.966$ & This study \\
\hline
\end{tabular}

When the monthly changes of total hardness values were examined according to Karasu stream parameter results, the lowest value was determined at $\mathrm{K} 4$ station in February $(10.10 \mathrm{mg} / \mathrm{L})$ and the highest value was found at K3 station in July $(429.10 \mathrm{mg} / \mathrm{L})$. Total hardness predominantly comes from dissolved calcium or magnesium salts, the salinity of which is carbonate, phosphate, chloride, sulphate, nitrate and silicates of these elements (Şengül and Türkman, 1998). For this reason, it is known that the geological structure plays an important role in the total hardness value of water resources. The annual average total hardness value of Karasu stream was determined as $130.84 \mathrm{mg} / \mathrm{L}$. The high average value of total hardness is an indication that the geological structure of the basins of water resources is rich in calcium and magnesium minerals. When the total hardness grades of four stations are examined, minimum values were detected in February (winter) and maximum values were found in April (spring). This result can be explained as an indication of the passage of mineral substances from the continental areas to the rivers through rain water.

Chemical oxygen demand (COD) is a measure of the amount of oxygen needed to oxidize the substances present in the water with available oxidizable properties. 
Oxidation takes place on the basis of redox reactions. During our study, the COD values of Karasu Stream were determined at $6.84-22.12 \mathrm{mg} / \mathrm{L}$. As is seen, these values are in parallel with the changes in the dissolved oxygen value considering COD values in the water source. Months of increase in the amount of dissolved oxygen showed an increase in COD values. Comparing the values of the present study are compared with the values of other studies, it can be said that the results are different. The reason is regional changes and differences in environmental impact.

Phosphorus emerges in forms of phosphate in natural waters. These forms are organic phosphate, orthophosphate and condensed phosphates. These forms of phosphates enter the water resources in different ways. The monthly average $\mathrm{PO}_{4}^{-3}-\mathrm{P}$ values of Karasu stream were found to be between 0.006 and $0.055 \mathrm{mg} / \mathrm{L}$. The $\mathrm{PO}_{4}^{-3}-\mathrm{P}$ values of the water source show fluctuations on a monthly basis at the stations. This may be indicated as a source of fluctuations, depending on the locations of the stations, the chemical content of the geological structure, agricultural activities, organic activities in the water and wastes of urban detergents and derivatives. When the phosphorus concentrations of the four stations were examined, the minimum values were found in November (excluding K2) and the maximum values were found in May. The increase in vegetable production in agricultural areas, which offers the lowest value of phosphorus in November, can be explained by the fact that the acquired chemicals have been used by plants and that fertilizing activities are low. Phosphorus growth in May may be interpreted as the result of intensive fertilization activities in May and early May, with a surplus of rainfall in this period or irrigation water as a result of transporting chemical substances from agricultural areas to natural waters. It also strengthens the possibility of human activity increase and the release of urban detergent wastes in the region. The results of the study show that the $\mathrm{PO}_{4}^{-3}$ $\mathrm{P}$ values are within the range of $\mathrm{PO}_{4}^{-3}-\mathrm{P}$ values realized in different areas. According to the European Union Water Framework Directive, total phosphorus value is envisaged to be in the range of $0.01-0.07 \mathrm{mg} / \mathrm{L}$ (EC, 2000a). In the present study, the values of $\mathrm{PO}_{4}^{-3}-\mathrm{P}$ do not exceed the specified values and the amount of phosphorus added to the water source is not too much.

As a nutrient like nitrogen, phosphorus elements come from domestic and industrial wastes, from erosion, from agricultural soils through drainage water and rainwater. The final result of reaching the maximum level in the lake, river, stagnant water and the gulf causes excessive algal growth and finally leads to eutrophication problem. When monthly changes of total nitrogen values of Karasu Stream are evaluated, the minimum with total nitrogen level of $0.122 \mathrm{mg} / \mathrm{L}$ was found in May at the K4 station and the maximum with value of $0.974 \mathrm{mg} / \mathrm{L}$ was measured in February at the K1 station (Table 3).

According to the station and season changes were investigated. When monthly changes of anionic detergent values of Karasu Stream are examined, the minimum with anionic detergent level of $0.005 \mathrm{mg} / \mathrm{L}$ was measured in February at the K2 station and the maximum with value of $0.973 \mathrm{mg} / \mathrm{L}$ was found in August at the K3 station. (Table 4). The reasons for setting the minimum value in
February are less population density and human activities. Sinop is a seaside city and there is an increase in population due to tourism seasonality in August. The reasons for determining the maximum value in August, depending on the population density, is likely to strengthen the effectiveness of the increase in human activity.

When the value of anionic detergent was evaluated seasonally, a minimum level of $0.107 \mathrm{mg} / \mathrm{L}$ was detected in the spring season and a maximum level of $0.703 \mathrm{mg} \mathrm{L}$ was found in the autumn season. When the anionic detergent level is analyzed according to the stations, it is seen that the average values are higher in summer and autumn months. The determination of the maximum values of four stations (K1 0.559 ; K2 0.571; K3 0.628; $\mathrm{K} 40.703 \mathrm{mg} / \mathrm{L}$ ) in the autumn season indicates that the amount of detergent supplied to the rivers from continental areas is greater than other times. The minimum values were measured at following stations and seasons; it was determined that station K1 0.107 and K3 $0.139 \mathrm{mg} / \mathrm{L}$ in the spring season, station $\mathrm{K} 20.120$ and $\mathrm{K} 4$ $0.119 \mathrm{mg} / \mathrm{L}$ in the winter season. The amount of anionic detergent concentration of Karasu stream in a large amount of spring and winter precipitation is greatly reduced (Table 4). In addition, it cannot be a detergent accumulation in water since the amount of detergent supplied to the rivers from terrestrial areas is low, due to the possibility of deterioration of the present detergent (Ivanković, and Hrenović, 2010) and the rivers are flowing water.

Biodegradation refers to the microbial degradation of organic substances (EC, 2004; Altuğ, 2005). Primary biodegradation refers to the structural change (transformation) of a surfactant by microorganisms resulting in the loss of its surface-active properties due to the degradation of the parent substance and consequential loss of the surface-active property. Ultimate biodegradation refers to the level of biodegradation achieved when the surfactant is totally used by microorganisms resulting in its breakdown to inorganic endproducts such as carbon dioxide, water and mineral salts of any other elements present (mineralization) and new microbial cellular (biomass) constituents (MerrettigBruns, and Jelen, 2009).

At K1 station, the minimum anionic detergent level was measured in April with $0.093 \mathrm{mg} / \mathrm{L}$, and the maximum value was measured in November with 0.823 $\mathrm{mg} / \mathrm{L}$. The minimum value of phosphorus was determined with $0.018 \mathrm{mg} / \mathrm{L}$ in November and the maximum value with $0.055 \mathrm{mg} / \mathrm{L}$ in May. The minimum level of phosphorus in the month of November and the maximum level of anionic detergent strengthens the possibility of a negative correlation between these two forms. If the temperature of the winter is too low, it can cause phytoplankton deaths. In case the season shifts towards the spring, the possibility of degradation of organic materials (mass mortality and live residues) is strengthened in parallel with the increase in temperature. For this reason, the phosphorus or phosphates in the organic matter passes into the water and the concentration of phosphorus in the water may increase. This clearly demonstrates why the phosphorus concentration is minimum in winter and maximum in spring. Kivrak 
(2011) found that a significant part of the phytoplankton development was in spring and that the concentration of phytoplankton was very low in other seasons when he studied in Karamuk Lake. He also noted a reduction in phytoplankton concentrations at the end of autumn and winter. In addition, he emphasized that the phytoplankton density is rather low in these periods when water temperature and daylight decrease. This shows that phosphorus, which reaches the maximum level in the spring and has nutritive element properties, is reduced as a result of being consumed by organisms (plankton, bacteria, fish, etc.) and decreases to a minimum level in summer. At other stations (K2, K3 and K4), it can be said that similar changes and occurrences occur for the same reasons (Table 2).

The correlation between all obtained physicochemical parameter (anionic detergent, P, TN, COD, DO and temperature concentrations) values is shown in Table 6. Although the correlation between anionic detergent and phosphorus and total nitrogen is very significant $(\mathrm{P} \leq 0,001)$, a negative relationship is found $(\mathrm{r}=-0.320, \mathrm{r}=$ -0.318 , respectively). This is because phosphorus and nitrogen may not necessarily increase when the detergent concentration is increasing in the water environment. Moreover, phosphorus and nitrogen may become miscible in the water after detergent degradation, and a certain amount of time is required. It is known that the detergent's degradation can take place if physicochemical and biological conditions are appropriate in the same process. In addition, phosphorus, which is effective in the growth of algae, was reported to be ingested by detergents at the rate of 7\% (Folsom and Oliver, 1980). Y1lmaz et al. (2006) explained that there are different biological and physical influences on phosphate and detergent related to time in their research on İzmir Gulf. It is also known that the nitrogen cycle is dependent on the time of biochemical and physical effects.

In our study, the correlation between DO and phosphorus in water ecosystem is very significant $(\mathrm{P} \leq 0.001)$ and negative $(\mathrm{r}=-0.292)$. Oxygen is a molecule that constantly enters the water with physical (with waves of water from the atmosphere) and biochemical effects (with photosynthesis of phytoplankton). Phosphorus is consumed by aquatic organisms as a food source. The lack of phosphorus in the environment, where there is a lot of oxygen, indicates that there is an inverse relationship between the amount of phosphorus and DO. This result supports the possibility that phosphorus has been consumed by organisms. According to our study findings, chemical oxygen demand (COD) is the amount of oxygen used during the breakdown of natural and contaminating organic loads with strong chemical oxidizers. Correlation between COD and phosphorus is very significant $(\mathrm{P} \leq 0.001)$ and negative $(\mathrm{r}=-0.650)$. As is DO, if the organic compounds are concentrated in the aquatic environment, the COD is very high. Phosphorus is an element consumed by organisms in the aquatic environment because it is a nutrient element. Therefore, it is a natural fact that the phosphorus concentration is low. Since the solubility of anionic detergents is very high and easy to degrade, the chemical oxygen demand is very high in their environment. For this reason, the correlation between KOI and detergent is very significant $(\mathrm{P} \leq 0.001)$ and positive $(\mathrm{r}=0.576)$. Microorganisms can decompose or reduce hydrocarbons and detergents using enzymes in nature. Nitrogen and phosphorus present in the contents of organic compounds may pass into the water. For this, time and conditions must be appropriate (Table 6).

Table 6 Correlation between Anionic Detergent, P, TN, COD, DO and Temperature values in Stations.

\begin{tabular}{|c|c|c|c|c|c|}
\hline Parameters & A. Detergent & $\mathrm{P}$ & $\mathrm{TN}$ & COD & DO \\
\hline $\mathrm{P}$ & $-0.320 * *$ & & & & \\
\hline TN & $-0.318 * *$ & 0.146 & & & \\
\hline COD & $0.576 * *$ & $-0.650 * *$ & -0.159 & & \\
\hline DO & -0.008 & $-0.292 * *$ & 0.090 & 0.157 & \\
\hline Temperature & -0.038 & $0.235^{*}$ & -0.086 & $-0.327 * *$ & $-0.818 * *$ \\
\hline
\end{tabular}

$\mathrm{P} \geq 0.05 ;(*) \mathrm{P} \leq 0.01 ;(* *) \mathrm{P} \leq 0.001$

Table 7 Result of physicochemical parameters of Karasu Stream according to inland water resources classes.

\begin{tabular}{|c|c|c|c|c|}
\hline \multirow{2}{*}{ Water quality parameters } & \multicolumn{4}{|c|}{ Water quality Classes } \\
\hline & Class I & Class II & Class III & Class IV \\
\hline $\mathrm{pH}$ & $\mathrm{X}$ & $\mathrm{X}$ & & \\
\hline Temperature & $X$ & & & \\
\hline DO & & $\mathrm{X}$ & $\mathrm{X}$ & \\
\hline COD & $\mathrm{X}$ & & & \\
\hline $\mathrm{P}$ & & $X$ & & \\
\hline $\mathrm{TN}$ & & $X$ & & \\
\hline A. Detergent & & $\mathrm{X}$ & $\mathrm{X}$ & \\
\hline
\end{tabular}

\section{Conclusion}

In the change of anionic detergent and physicochemical parameter values; it can be said that it is the effect of precipitation, melting of snow, and evaporation depending on temperature, and most importantly environmental effects, which are agricultural activities, industrial wastes, urban cleaning materials and derivatives. The water quality class of Karasu Stream was evaluated according to inland water resources classes (SWQR, 2015) and it was summarized in Table 7. The $\mathrm{pH}$ value has been determined as class $\mathrm{I}$ in stream water, 
whereas the temperature and COD values are class II. Waters of Class-I are a high quality water and potential drinking water. It is suitable for many purposes such as trout and animal breeding, swimming and so on. Water is equivalent to Class II in terms of phosphorus and total nitrogen concentrations but is equivalent to Class II and III in terms of anionic detergent concentration and DO values. However, according to the quality criteria of the European Environment Agency's flotation water quality, it is close to the concentration $(0.3 \mathrm{mg} / \mathrm{L})$ of the surface active agent stained with methylene blue (EEC, 2003). Water of Class-II quality is surface water with slightly contaminated and potentially potable. For recreational purposes; it can be used for fish production outside the trout and as irrigation water provided that irrigation water quality criteria are met. Class-III water is polluted water that can be used as aquaculture and industrial water after an appropriate treatment, but never for food and textile purposes.

It has been determined that the concentration values of some of the parameters of the study which we have carried out at Karasu Stream are at high values. This strengthens the possibility that eutrophication may begin or increase in the water source. In order to ensure the sustainability of water resources; water resources conservation, improvement and taking the necessary measures to leave the next generation in the best condition, the seriousness and the importance of the issue must be underscored. The main objective of the European Union Water Framework Directive is; to spread the concept of management based on river basin (EC, 2000b). With these steps; particularly, prevention and development of further degradation of water environments and long-term preservation of existing water resources are required. Çiçek et al. (2015) explained that in total 153 fish species are endemic in Turkish fresh water habitats. Moreover, they emphasized that they constitute $41.58 \%$ of total fish species. For this reason, the quality of inland waters is important for endemic species to maintain their vital activities and not to be destroyed from nature. In addition, our results will be a source for future research.

\section{Acknowledgement}

The data of the project "Investigation of anionic detergent pollution in Karasu river and Sırakaraağaçlar stream" were used (SÜF-1901.13-05). Master thesis work has been done from project data. We would like to thank Sinop University Scientific Research Project Coordination Unit for their contribution.

\section{References}

Akkan T. 2017. An Assessment of Linear Alkylbenzene Sulfonate (LAS) Pollution in Harsit Stream, Giresun, Turkey. Fresenius Environmental Bulletin, 26(5): 32173221. Available from: http://www.prt-parlar.de/ download_feb_2017/5

Altuğ G. 2005. Bacteriological marine pollution. In: Güven KC. and Öztürk B. (Eds.), Marine Pollution, Basic Pollutants and Methods of Analysis. pp:225-275, TÜDAV Publication, 21: 499pp. Available from http://www.tudav.org/ images/2014/new/pdfs/deniz_kirliligi.pdf
APHA. 1981. Standard Methods for the Examination of Water and Wastewater, 15th ed.; APHA (American Public Health Association): Washington, D.C, USA, 85-99, 773-779, 786828pp.

APHA. 1965. Standard methods for the examination of water and waste water. 19th edition. American Public Health Association Inc., New York, 1193 pp.

Bakan G, Şenel B. 2000. Research on Bottom Sediment and Water Quality of Samsun-Mert Stream at the Discharge into the Black Sea. Turkish Journal of Engineering and Environmental Sciences, (24): 135-141. Available from: http://journals.tubitak.gov.tr/engineering/issues/muh-00-243/muh-24-3-2-98028.pdf

Bat L, Akbulut M, Çulha M, Sezgin M. 2000. The Macrobenthic Fauna of Sırakaraağaçlar Stream flowing into Black Sea at Akliman Sinop. Turkish Journal of Marine Sciences, 6(1): 71-86. Available from: http://www.blackmeditjournal.org/ pdf/2000_vol6_no1-7.pdf

Bayram A, Önsoy H. 2011. Determination of the polluters carried by the stream Harşit (Giresun - Tirebolu) into the Black Sea. 7th Coastal Engineering Symposium (pp. 545555). Trabzon, Turkey. Available from: http://www.imo.org.tr/resimler/ekutuphane/pdf/16832_48_2 9.pdf

Boztuğ D, Dere T, Tayhan N, Yıldırım N, Danabaş D, Yıldırım NC, Önal AÖ, Danabaş S, Ergin C, Uslu G, Ünlü E. 2012. Physico-Chemical Characteristics of Uzuncayir Dam Lake (Tunceli) and Water Quality. Adıyaman Üniversity, Journal of Science, 2 (2): 93-106. Available from: http://dergipark.ulakbim.gov.tr/adyuebd/article/view/500007 9679/5000074045

Bulut C, Akçimen U, Uysal K, Küçükkara R, Savaşer S. 2010. Seasonal variations of physicochemical and microbiological parameters of the Karanfilliçay creek water and its aquacultural evaluation. Journal of the Institute of Science and Technology of Dumlupınar University, (21): 1-8. Available from: https://birimler.dpu.edu.tr/app/views/panel/ ckfinder/userfiles/16/files/Dergiler/21/sayi1pdf.pdf

Bulut C, Akçimen U, Uysal K, Küçükkara R, Savaşer S, Tokatlı C, Öztürk GN, Köse E. 2012. Water qality of Kestel creek (Burdur) and assesment for salmonid culture. Journal of the Institute of Science and Technology of Dumlupinar University, (28): 1-10. Available from: https://birimler.dpu.edu.tr/app/views/panel/ckfinder/userfiles /16/files/Dergiler/28/fbe-13.pdf

Cirik S, Cirik Ş. 2008. Limnology. Ege University, Faculty of Fisheries Press, Izmir, Turkey, $166 \mathrm{pp}$.

Cook R. 1968. The bacterial degradation of synthetic anionic detergents. Water Research, 2 (12): 849-866. DOI: https://doi.org/10.1016/0043-1354(68)90039-0

Çarlı U. 2015. Investigation of some physico-chemicaland microbiological parameters in Karasu and Sırakaraağaçlar stream (Master's Thesis). Sinop University, Sinop, Turkey.

Çiçek E, Birecikligil SS, Fricke R. 2015. Freshwater fishes of Turkey: a revised and updated annotated checklist. Biharean biologist, 9(2): 141-157. Available from: http://biozoojournals.ro/ bihbiol/cont/v9n2/bb_151306_Cicek.pdf

Çiçek NL, Ertan ÖO. 2012. Determination of the water quality of Köprüçay river (Antalya) according to the physicochemical parameteres (Antalya). Ecology, 21 (84): 54-65. Available from: http://uvt.ulakbim.gov.tr/uvt/index.php? cwid=9\&vtadi=TPRJ\%2CTTAR\%2CTTIP\%2CTMUH\%2C TSOS\%2CTHUK\&ano=161068_1cb8a5bb038c2a242e79a8 $36 \mathrm{f} 57 \mathrm{e} 6 \mathrm{a} 74$

Dinçer S. 2014. Determination of the Water Quality and Pollution Level of Çanakçı Creek (Master's Thesis). Giresun University, Giresun, Turkey. Available from: http://acikerisim.giresun.edu.tr/xmlui/bitstream/handle/1234 $56789 / 48 /$ Serhat $\% 20$ Din $\%$ C3\%A7er.pdf? sequence $=1$ 
EC. 2000a. Common Implementation Strategy for the Water Framework Directive (2000/60/EC: European Commission), Report No: 30. European 327pp. Available from: https://circabc.europa.eu/sd/a/9060bdb4-8b66-439e-a9b0a5cfd8db2217/Guidance_document_23_Eutrophication.pdf

EC. 2000b. Directive 2000/60/EC (European Commission) of the European Parliament and of the Council of 23 october 2000. Establishing a framework for community action in the field of water policy. Official Journal of the European Communities I. 327:1-73pp. Available from: http://eurlex.europa.eu/resource.html?uri=cellar:5c835afb-2ec6-4577bdf8-756d3d694eeb.0004.02/DOC_1\&format=PDF

EC. 2004. EC No 648/2004 of the European Parliament and of the Council of 31 March 2004 on detergents. In Official Journal of the European Union, 08 April 2004, 1-35pp. Available from: http://eur-lex.europa.eu/legal-content/ en/TXT/PDF/?uri=CELEX:02004R064820150601\&from $=\mathrm{EN}$

EEC. 2003. The Bathing Water Regulations (LN380/2003) which transposes the European Bathing Water Directive CD76/160/EEC (European Environment Commission). Report on monitoring of physico-chemical parameters, Malta Environment and Planning Authority, April 2006. Available from: https://era.org.mt/en/Documents/Bathing_ Water_Quality_Monitoring-FINAL_24.4.2006.pdf

Egemen Ö. 2000, Environment and Water Pollution, İzmir, Turkey, EÜFF Press., 120pp.

Egemen Ö, Sunlu U. 1996. Water Quality, Ege University, İzmir, Turkey, EÜFF Press., 153pp.

Er CB. 2014. Some physicochemical and microbiological properties of drinking water in Kilis province (Master's Thesis). Kilis 7 Aralık University, Kilis, Turkey. Available from: http://fbe.kilis.edu.tr/dosyalar/belgeler/Cem\%20Baran $\% 20$ Er.pdf

Gültekin F, Ersoy AF, Hatipoğlu E, Celep S. 2012. Determination of Water Quality Parameters in Wet Season of Surface Water in Trabzon. Ecology, 21 (82): 77-88. Available from: http://uvt.ulakbim.gov.tr/uvt/index.php? cwid=9\&vtadi=TPRJ\%2CTTAR\%2CTTIP\%2CTMUH\%2C TSOS\%2CTHUK\&ano=143997_c626afac9c444b7e63c7a81 b9d3e6e51

Gündoğdu A. 1995. Investigation of Anionic Detergents Pollution in shore of Sinop (Master's thesis). Ondokuz Mayis University, Samsun, Turkey. Available from: http://acikerisim.sinop.edu.tr:8080/xmlui/handle/11486/884

Gündoğdu A., Erdem M, 1995. Investigation of anionic detergent pollution in shore of Sinop. II. National Ecology and Environment Congress, September 11-13, 1995 , Ankara, 611-624. Available from: https://scholar.google.com.tr/citations?user=IolJ06CIOgIC\& $\mathrm{hl}=\mathrm{tr}$

Gürel E. 2011. Determination of Porsuk Stream Water Quality (Master Thesis). Eskişehir Osmangazi University, Eskişehir, Turkey.

İleri R. 2000. "Çevre Biyoteknolojisi”, Değişim Yayınları, 50122, Adapazarı.

Ivanković T, Hrenović J. 2010. Surfactants in the environment. Arhiv za Higijenu Rada i Toksikologiju, 61: 95-110. DOI: https://doi.org/10.2478/10004-1254-61-2010-1943

Kivrak E. 2011. Seasonal variations in phytoplankton community and some physico-chemical features of Lake Karamuk (Afyonkarahisar-Turkey) Ege Journal of Fisheries and Aquatic Sciences. 28: 1: 9-19. Available from: http://www.egejfas.org/download/article-file/57364

Kivrak E, Uygun A, Kalyoncu H. 2012. Application of Diatom Indices to Assess Water Quality of the Akarçay Stream (Afyonkarahisar, Turkey). Afyon Kocatepe University Journal of Sciences, 12: 27-38. Available from: http://fenbildergi.aku.edu.tr/1202/021003(27-38)(OS001).pdf
Kundu S, Coumar MV, Rajendiran S, Ajay SR, Rao AS. 2015. Phosphates from detergents and eutrophication of surface water ecosystem in India. Current Science. 108 (7): 13201325.

http://www.currentscience.ac.in/Volumes/108/07/1320.pdf

Kurnaz A, Mutlu E, Aydın Uncumusaoğlu, A. 2016. Determination of Water Quality Parameters and Heavy Metal Content in Surface Water of Çiğdem Pond (Kastamonu/Turkey). Turkish Journal of Agriculture - Food Science and Technology, 4(10): 907-913. DOI: https://doi.org/10.24925/turjaf.v4i10.907-913.942

Leithe W. 1973. The analysis of organic pollutants in water and waste water. Translator: STS, Inc., Consulting technical editor: Nina McClelland, Ann Arbor Science Publishers, Ann Arbor, Michigan, USA, 213 pp.

Merrettig-Bruns U, Jelen E. 2009. Anaerobic Biodegradation of Detergent Surfactants. Materials, 2: 181-206. DOI: https://doi.org/10.3390/ma2010181

Minareci O. 2007. Investigation of detergent pollution in the Gediz river (PhD Thesis). Celal Bayar University, Manisa, Turkey.

Minareci O, Öztürk M, Egemen Ö, and Minareci E. 2009. Detergent and phosphate pollution in Gediz river, Turkey. African Journal of Biotechnology, 8(15), 3568-3575. DOI: 10.5897/AJB09.167. Available online at: http://www.academicjournals.org/AJB

Morse GK, Lester JN, Perry RT. 1993. The Economic and Environmental Impact of Phosphorus Removal from Wastewater in the European Community, Selpher Press., London, England, 1991pp.

Mutlu E, Kutlu B, Demir, T. 2016. Assessment of Çınarli Stream (Hafik -Sivas)'S Water Quality via PhysicoChemical Methods. Turkish Journal of Agriculture - Food Science and Technology,4(4): 267-278. DOI: http://www.agrifoodscience.com/index.php/TURJAF

Mutlu E, Kurnaz, A. 2017. Determination of seasonal variations of heavy metals and physicochemical parameters in Sakiz Pond (Kastamonu-Turkey). Fresen. Environ. Bull. 26(4), 2807-2816.

Oner O, Celik A. 2011. Investigation of some pollution parameters in water and sediment samples collected from the lower Gediz river basin. Ecology, 20 (78): 48-52. Available from: http://uvt.ulakbim.gov.tr/uvt/index.php? cwid=9\&vtadi=TPRJ\%2CTTAR\%2CTTIP\%2CTMUH \%2C TSOS\%2CTHUK\&ano=127577_f413aee589dcbb49cd9c04 80cefe 1026

Polat N, Akkan T. 2016. Assessment of Heavy Metal and Detergent Pollution in Giresun Coastal Zone, Turkey. Fresenius Environmental Bulletin, 25 (8): 2884-2890. Available from: http://www.prt-parlar.de/ download_ feb_2016/8

Şengül F, Türkman A. 1998. Water and Wastewater Analysis. TMMOB Chamber of Environmental Engineers Press, Izmir, Turkey, 152pp.

Şengün E. 2013. Determination of The Water Quality and Pollution Level of Aksu Creek (Master Thesis). Giresun University, Giresun, Turkey. Available from: http://acikerisim.giresun.edu.tr/xmlui/bitstream/handle/1234 56789/368/ERHAN\%20\%C5\%9EENG\%C3\%9CN.pdf?seq uence $=1 \&$ isAllowed $=\mathrm{y}$

Taşdemir M, Göksu ZL. 2001. Some Water Quality Features of Asi River (Asi Nehri'nin (Hatay Türkiye) Bazı Su Kalite Özellikleri). Ege Journal of Fisheries and Aquatic Sciences. 18 (1-2): 55-64. Available from: http://www.egejfas.org/ download/article-file/58087

Tepe Y. 2009. Determination of the Water Quality of Reyhanl Yenisehir Lake (Hatay). Ecology, 18, 70, 38-46. Available from: http://uvt.ulakbim.gov.tr/uvt/index.php?cwid=9\&vtadi $=$ TPRJ $\% 2$ CTTAR $\% 2$ CTTIP\%2CTMUH $\% 2$ CTSOS $\% 2 \mathrm{CTH}$ UK\&ano=98005_5aabbdcb93cd34e514717c3665d0b831 
Tepe Y, Mutlu E. 2004. Physico-Chemical Properties of Water Resource in Hatay Harbiye (Hatay Harbiye Kaynak Suyunun Fizikokimyasal Özellikleri). Journal of the Institute of Science and Technology of Dumlupınar University, 6: 77-88. Available from: http://birimler.dpu.edu.tr/app/views/ panel/ckfinder/userfiles/16/files/Dergiler/6/07.pdf

TSI. 2005. Drinking and Use Water Standards, Turkish Standards Institute (Türk Standartları Enstitüsü, İçme ve Kullanma Suları Standartları). Available from: http://www.opalsu.com.tr/mevzuat/Havuzsularininozonlanm asiTSE_11899.pdf

Uzun S, Uzun A, Yılmaz C, Zeybek Hİ. 2005. Erfelek Waterfalls, Sinop. Eastern Geographical Review, 10/14, 331-348. Available from: http://e-dergi.atauni.edu.tr/ ataunidcd/article/view/1021006265/1021005969

SWQR. 2015. Surface Water Quality Regulation. Legislation information system in Turkey. Official Gazette dated 15.04.2015 and numbered 29327. Available from: https://kms.kaysis.gov.tr/Home/Goster/41792?AspxAutoDet ectCookieSupport=1
Verep B, Serdar O, Turan D, Şahin C. 2005. Determination of Water Quality in terms of Physico-Chemical Structure of the River Iyidere (Trabzon). Ecology, 14(57): 26-35. Available from: http://uvt.ulakbim.gov.tr/uvt/index.php?cwid=9\& vtadi=TPRJ $\% 2$ CTTAR $\% 2$ CTTIP $\% 2$ CTMUH $\% 2$ CTSOS $\% 2$ CTHUK\&ano=54502_d2442e87d5c3e5cdcc689aa27c053f51

Yildiz İ. 2013. Determination of the Water Quality and Pollution Level of Gelevera Creek (Master Thesis). Giresun University. Giresun, Turkey. Available from: http://acikerisim.giresun.edu.tr/xmlui/bitstream/handle/1234 56789/69/\%C4\%B0SMA\%C4\%B0L\%20YILDIZ.pdf?seque nce $=1 \&$ is Allowed $=$ n

Yilmaz Ö, Sunlu U, Sunlu FS. 2006. Investigation of Anionic Detergent Levels in İzmir Gulf (İzmir Körfezi'nde Anyonik Deterjan Düzeylerinin Araştırılması). Ege Journal of Fisheries and Aquatic Sciences. 23(1-2): 107-111. Available from: http://www.egejfas.org/download/article-file/57596 